J. Phys. IV France 130 (2005) 133-139

(C) EDP Sciences, Les Ulis

DOI: $10.1051 / \mathrm{jp} 4: 2005130009$

\title{
Influence du solvant sur la dynamique interne de la butyrylcholinestérase et sur la dynamique de l'eau d'hydratation : une étude par diffusion élastique incohérente de neutrons
}

\author{
F. Gabel
}

\author{
Institut de Biologie Structurale, 41 rue Jules Horowitz, 38027 Grenoble, France \\ Actuellement EMBL, Meyerhofstrasse 1, 69117 Heidelberg, Germany
}

\begin{abstract}
Résumé. L'enzyme butyrylcholinestérase humaine a été étudiée par diffusion élastique incohérente de neutrons entre 20 et $285 \mathrm{~K}$ sous différentes conditions de solvant. La dynamique globale sur une échelle de l'Ångström et de la nanoseconde, exprimée en déplacements carrés moyens, a été déterminée dans le cadre de l'approximation Gaussienne. La comparaison d'échantillons lyophilisés de différents tampons et hydratés en $\mathrm{H}_{2} \mathrm{O}$ ou en $\mathrm{D}_{2} \mathrm{O}$ a donné les résultats suivants: 1) La dynamique globale interne de l'enzyme varie en fonction du type de sel présent dans le solvant. 2) La dynamique de l'eau d'hydratation est différente en fonction de la température et de la dynamique interne de l'enzyme.
\end{abstract}

\section{INTRODUCTION}

La relation importante entre l'environnement moléculaire d'une protéine et sa dynamique interne a été soulignée par plusieurs expériences de diffusion de neutrons en poudre ou solution dans les dernières années [1]. Notamment le degré d'hydratation et la composition du solvant ont été utilisés comme paramètres expérimentaux. La transition dynamique des protéines autour de $200 \mathrm{~K}$, qui a été montrée comme étant liée à une activité biologique dans certains cas, nécessite une hydratation suffisante [2]. L'addition des cryo-protectants, comme le glycérol [3] ou le tréhalose [4] réduit sensiblement la flexibilité interne des protéines et supprime la transition dynamique. Bien qu'étant un paramètre biologique important pour la stabilité structurale des protéines, la composition saline du solvant a été peu étudiée dans les expériences de neutrons.

Les expériences de diffusion incohérente élastique de neutrons (IENS) sont généralement conduites en présence de $\mathrm{D}_{2} \mathrm{O}$ en raison de la faible contribution de l'eau lourde à la diffusion incohérente totale. Ainsi, très peu d'informations sur la dynamique propre de l'eau d'hydratation sont disponibles par cette méthode, notamment en fonction de la composition du solvant. Pourtant, la dynamique du solvant a été proposée pour influencer directement la dynamique interne des protéines [5-7].

L'article ci-présent aborde deux aspects: 1) Les relations entre la composition saline du solvant et la dynamique interne d'une enzyme, la butyrylcholinestérase humaine (HuBChE) et 2) la capacité de l'IENS à étudier la dynamique de l'eau d'hydratation. Afin d'étudier ces deux domaines, une série d'expériences IENS sur la $\mathrm{HuBChE}$ en forme de poudre hydratée en $\mathrm{H}_{2} \mathrm{O}$ et en $\mathrm{D}_{2} \mathrm{O}$ a été effectuée. Les résultats montrent: 1) une forte dépendance entre la dynamique interne de la HuBChE et la nature du sel présent dans le solvant et 2) la possibilité de discriminer, en fonction de la température, entre la dynamique interne de l'enzyme et celle de l'eau d'hydratation. Ces résultats sont discutés dans le cadre restreint de la stabilité de la $\mathrm{HuBChE}$ en fonction du tampon et dans le cadre général des interactions entre protéines et l'eau d'hydratation. 


\section{MATÉRIEL ET MÉTHODES}

\subsection{Préparation des échantillons de $\mathrm{HuBChE}$}

L'enzyme a été purifiée selon un protocole [8] à partir du plasma humain. La procédure comprend des étapes successives: filtration, ajustement $\mathrm{du} \mathrm{pH}$, adsorption de batch et/ou de la chromatographie de colonne de procainamide-sépharose, suivi par une chromatographie d'échange d'ions. L'enzyme ainsi purifiée a été stockée dans un tampon $10 \mathrm{mM}$ phosphate de sodium à $\mathrm{pH} 8$. Pour les expériences de diffusion neutronique, les échantillons ont été préparés de la manière suivante:

" $\mathrm{HuBChE}-\mathrm{H}_{2} \mathrm{O}$ ": HuBChE dialysée contre $25 \mathrm{mM}$ d'acétate d'ammonium volatile à $\mathrm{pH} 8$. Après plusieurs jours de séchage sur gel de silice, l'échantillon a été hydraté à 0.44 grammes de $\mathrm{H}_{2} \mathrm{O}$ par gramme de matériel sec (dénoté $0.44 \mathrm{~g} / \mathrm{g}$ ) par échange de vapeur.

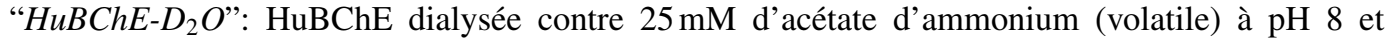
hydratée en $\mathrm{D}_{2} \mathrm{O}$ à $0.47 \mathrm{~g} / \mathrm{g}$.

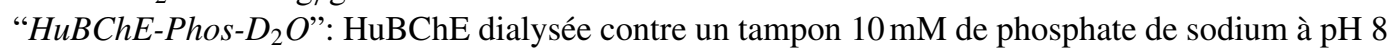
et hydratée à $0.46 \mathrm{~g} / \mathrm{g}$ en $\mathrm{D}_{2} \mathrm{O}$.

"HuBChE-Tris- $\mathrm{D}_{2} \mathrm{O}$ ”: HuBChE dialysée contre un tampon $10 \mathrm{mM}$ de Tris- $\mathrm{HCl}$ (non-volatile) à $\mathrm{pH}$ 8 et hydratée à $0.43 \mathrm{~g} / \mathrm{g}$ en $\mathrm{D}_{2} \mathrm{O}$.

Pour $\mathrm{HuBChE}-\mathrm{H}_{2} \mathrm{O}$, l'eau d'hydratation contribue pour environ $40 \%$ à la section efficace totale de l'échantillon [9]. Les volumes des tampons non-volatiles (phosphate de sodium et Tris-HCl) ont été ajusté afin d'établir une molarité d'ions de sel comparable pour tous les échantillons, de l'ordre de 1000 ions par monomère d'enzyme après lyophilisation [9]. L'activité enzymatique a été mesurée par le test d'Ellman [10] et les pertes d'activité enregistrées à l'issue des expériences de neutrons se sont révélées être inférieures à $20 \%$.

\subsection{La diffusion élastique incohérente de neutrons et l'influence de la résolution instrumentale}

En se limitant aux atomes d'hydrogène et en négligeant les contributions cohérentes, la loi de diffusion, qui est proportionnelle à la probabilité de mesurer un neutron sous un vecteur de diffusion $\mathbf{Q}$, s'écrit de la manière suivante:

$$
S(\mathbf{Q}, \omega)=\frac{1}{2 \pi} \int_{-\infty}^{+\infty} I(\mathbf{Q}, t) \exp (-i \omega t) d t
$$

avec la fonction intermédiaire de diffusion exprimée comme

$$
I(\mathbf{Q}, t)=\int_{-\infty}^{+\infty} G(\mathbf{r}, t) \exp (i \mathbf{Q} \cdot \mathbf{r}) d^{3} \mathbf{r}
$$

$G(\mathbf{r}, \mathrm{t})$ est la fonction d'auto-corrélation des atomes d'hydrogène et décrit la probabilité de trouver un atome d'hydrogène dans un point $\mathbf{r}$ au temps $t$ si le même atome était à l'origine au temps $0 . I(\mathbf{Q}, t)$ peut être séparée en deux composantes, respectivement indépendante et dépendante du temps [11]:

$$
I(\mathbf{Q}, t)=I(\mathbf{Q}, \infty)+I^{\prime}(\mathbf{Q}, t)
$$

Ainsi, la loi de diffusion théorique contient une partie indépendante du temps, le facteur de structure élastique incohérent (EISF), et une partie qui dépend des mouvements des atomes:

$$
S_{\text {theo }}(\mathbf{Q}, \omega)=I(\mathbf{Q}, \infty) \delta(\omega)+\frac{1}{2 \pi} \int_{-\infty}^{+\infty} I^{\prime}(\mathbf{Q}, t) \exp (-i \omega t) d t
$$


Sur un instrument réel, la loi de diffusion mesurée s'exprime par une convolution avec la résolution instrumentale $R(\omega)$ :

$$
S_{\text {meas }}(\mathbf{Q}, \omega)=S_{\text {theo }}\left(\mathbf{Q}, \omega^{\prime}\right) \otimes R\left(\omega-\omega^{\prime}\right)=\int_{-\infty}^{+\infty} S_{\text {theo }}\left(\mathbf{Q}, \omega^{\prime}\right) R\left(\omega-\omega^{\prime}\right) d \omega^{\prime}
$$

Dans le cas d'une mesure à un transfère d'énergie nulle ("scan élastique"), l'intensité élastique (EI) mesurée peut être exprimée de la manière suivante:

$$
S_{\text {meas }}(\mathbf{Q}, 0)=I(\mathbf{Q}, \infty) R(0)+\frac{1}{2 \pi} \int_{-\infty}^{+\infty} \int_{-\infty}^{+\infty} I^{\prime}(\mathbf{Q}, t) R\left(0-\omega^{\prime}\right) \exp \left(-i \omega^{\prime} t\right) d \omega^{\prime} d t
$$

L'EI se sépare ainsi en deux parties: une partie provenant de l'EISF qui ne dépend que de la valeur de la résolution instrumentale à $\omega=0$, et en une partie recouvrant les contributions quasi-élastiques. Ce second terme dépend fortement de la résolution instrumentale, et contribue au fait que l'EI et l'EISF ne sont pas équivalents. Si les mouvements moléculaires ou atomiques sont confinés, et si la partie dépendante du temps de l'équation (6) ne varie pas en fonction de $\mathbf{Q}$ ou s'avère négligeable par rapport à l'EISF, l'EI peut être exprimée par l'approximation Gaussienne:

$$
S_{\text {meas }}(\mathbf{Q}, 0) \approx \exp \left(-\frac{1}{6} Q^{2}\left\langle u^{2}\right\rangle\right)
$$

Cette approximation est similaire, par analogie mathématique, à l'approximation de Guinier [12] couramment utilisée en diffusion aux petits angles. Sa condition d'applicabilité s'exprime par:

$$
Q^{2}\left\langle u^{2}\right\rangle \leq 2
$$

Les déplacements carrés moyens (MSDs), $\left\langle u^{2}\right\rangle$, sont ici définis de manière à représenter les amplitudes des mouvements [13].

\subsection{L'instrumentation et l'extraction des MSDs}

Les expériences de diffusion ont été effectuées sur le spectromètre de rétro-diffusion IN16 à l'Institut Laue-Langevin à Grenoble, dans une gamme de température allant de 20 à $285 \mathrm{~K}$. La résolution en énergie utilisée, $\Delta \mathrm{E}=0.9 \mu \mathrm{eV}$, correspond à une résolution temporelle de l'ordre de $730 \mathrm{ps}$. La gamme de $Q^{2}$ utilisée s'étendait de 1.13 à $2.46 \AA^{-2}$. Les valeurs de transmission des échantillons variaient entre 0.9 et 0.95 , et une correction pour la diffusion multiple n'a pas été effectuée. Les signaux diffusés ont été corrigés de la diffusion du porte-échantillon et des corrections d'auto-absorption ont été réalisées par le programme FORTRAN 77 Iq0. Un enregistrement des profils de diffraction a été réalisé en parallèle aux mesures des « scans élastiques ». Des pics de Bragg correspondant à des micro-cristallites de glace et/ou de sel n'ont été observés pour aucun échantillon. Les intensités élastiques mesurées aux différentes températures ont été normalisées à la température la plus basse $(20 \mathrm{~K})$. Les MSDs ont été extraits de manière « classique » par la pente du logarithme de l'EI normalisée dans la gamme de $Q^{2}$ de 1.13 à $2.46 \AA^{-2}$.

\section{RÉSULTATS ET DISCUSSION}

\subsection{L'influence de la composition ionique du solvant sur la dynamique interne de l'enzyme}

Figure 1 montre, en fonction du solvant, les MSDs des échantillons $H u B C h E-P h o s-D_{2} O, H u B C h E$ Tris- $D_{2} O$ et $H u B C h E-D_{2} O$. Les MSDs de tous les échantillons augmentent de manière harmonique et se superposent jusqu'à environ $240 \mathrm{~K}$ où $H u B C h E-D_{2} O$ et $H u B C h E-T r i s-D_{2} O$ subissent une transition dynamique. $H u B C h E-P h o s-D_{2} O$, par contre, ne montre pas de transition dynamique à cette température, 


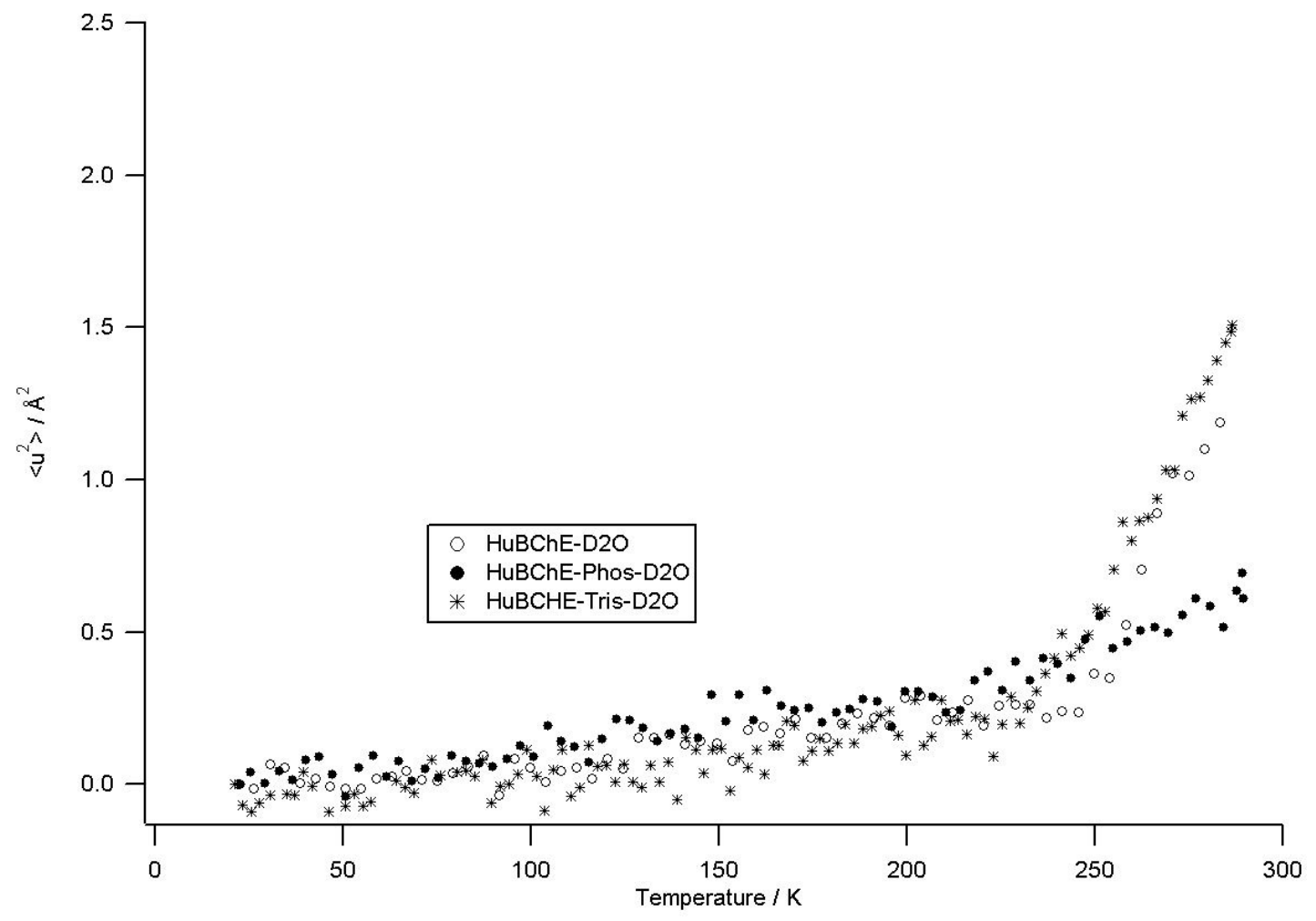

Figure 1. MSDs des trois échantillons hydratés en $\mathrm{D}_{2} \mathrm{O}$, en fonction de la température.

les MSDs augmentant de manière harmonique jusqu'à la température la plus haute mesurée $(285 \mathrm{~K})$. Tandis que la présence du Tris-HCl n'influence pas le comportement dynamique de l'enzyme dans la gamme de $\mathbf{Q}$ étudiée par rapport à l'échantillon sans sel $\left(H u B C h E-D_{2} O\right)$, la présence du phosphate de sodium supprime la transition dynamique et rapproche le comportement dynamique de l'échantillon à celui d'un échantillon sec (non représenté ici). L'effet du phosphate de sodium semble comparable avec les modifications engendrées sur la dynamique des protéines en présence des cryo-protectants comme le tréhalose [4]. La figure 1 montre que la modification de la dynamique interne de l'enzyme dépend du type ionique de soluté salin utilisé. Dans le cas étudié, l'effet du tampon suit la série de Hofmeister: le phosphate se trouve à pH 8 en majorité doublement chargé électriquement, et voit son action kosmotrope renforcée, c'est à dire influence fortement la dynamique et la structure de l'eau d'hydratation [14]. Le Tris- $\mathrm{HCl}$, quant à lui, se situe dans la gamme neutre-chaotrope de la série, les ions de sel influençant peu la dynamique et structure des molécules d'eau environnant l'enzyme. Les expériences présentées de diffusion neutronique ne peuvent pas renseigner sur le mécanisme microscopique intime responsable de la modification de la dynamique interne de l'enzyme. Cependant, les résultats obtenus sont en accord avec de précédents résultats [6], qui ont montré par simulation numérique qu'une réduction de la diffusion translationelle de l'eau d'hydratation entraîne une suppression de la dynamique interne d'une protéine hydratée. Une telle réduction de la dynamique de l'eau d'hydratation a été observée de manière indirecte [9] dans le cas de l'échantillon en phosphate de sodium mais pas dans l'échantillon sans sel. La restriction de la dynamique des molécules d'eau pourrait se transmettre à la protéine, d'une manière encore incomprise, à travers des liaison d'hydrogène de l'eau aux résidus de surface de la protéine. La restriction de la dynamique interne, en présence de l'agent kosmotrope constitué du phosphate de sodium, pourrait même fournir une explication de l'augmentation de la stabilité de la HuBChE observée en présence des ions kosmotropes [15]: la dénaturation, ne serait-ce que partielle 
de l'enzyme, nécessite des changements conformationnels importants. De telles trans-conformations seraient inhibées par l'incapacité de la protéine à subir une transition dynamique et ainsi à explorer un sous-espace conformationnel l'amenant vers l'état dénaturé.

\subsection{Comparaison de la dynamique interne des protéines et de l'eau d'hydratation en fonction de la température : échantillons hydratés en $\mathrm{D}_{2} \mathrm{O}$ et $\mathrm{H}_{2} \mathrm{O}$}

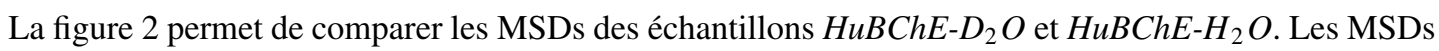
des deux échantillons sont identiques de 20 à environ $200 \mathrm{~K}$, en croissance linéaire, ce qui reflète la présence d'un potentiel harmonique pour tous les hydrogènes: les comportements dynamiques de l'eau d'hydratation et de la protéine ne peuvent être distingués dans cette gamme de température. A partir de $200 \mathrm{~K}$, les MSDs de $\mathrm{HuBChE}-\mathrm{H}_{2} \mathrm{O}$ dépassent nettement les MSDs de $H u B C h E-D_{2} O$ qui effectue une transition dynamique seulement à $250 \mathrm{~K}$. Au-delà de $260 \mathrm{~K}$, les MSDs de $H u B C h E-D_{2} O$ rejoignent les MSDs de $\mathrm{HuBChE}-\mathrm{H}_{2} \mathrm{O}$ et ce jusqu'à la température la plus haute mesurée $(285 \mathrm{~K})$. En ne se limitant qu'aux MSDs, la dynamique mesurée dans le cadre de l'approximation Gaussienne ne permet pas une séparation entre la dynamique des hydrogènes de la $\mathrm{HuBChE}$ et de l'eau d'hydratation à très basses températures $(20$ à $200 \mathrm{~K})$ et à très hautes températures $(260$ à $285 \mathrm{~K})$. Par contre, dans une gamme de température intermédiaire (de 200 à $260 \mathrm{~K}$ ), les contributions de l'eau d'hydratation $\mathrm{H}_{2} \mathrm{O}$ modifient le signal élastique par rapport à l'échantillon hydratée en $\mathrm{D}_{2} \mathrm{O}$.

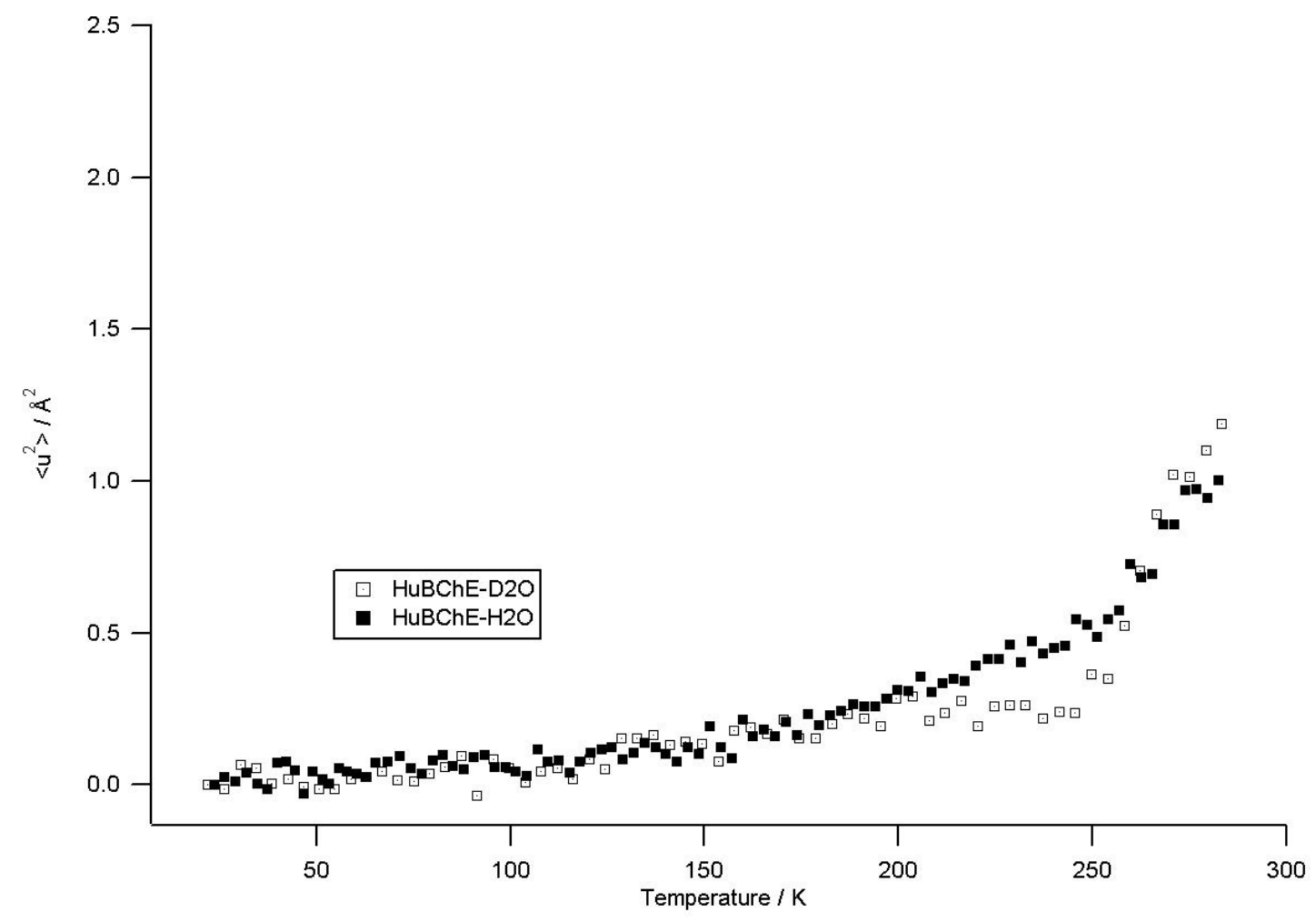

Figure 2. MSDs de la $\mathrm{HuBChE}$ hydratée en $\mathrm{H}_{2} \mathrm{O}$ et $\mathrm{D}_{2} \mathrm{O}$, en fonction de la température.

En supposant la dynamique interne de l'enzyme indépendante du type isotopique de l'eau d'hydratation, et en se limitant à une analyse du type « mouvements diffusifs » on peut décrire la situation de la manière suivante: entre $200 \mathrm{~K}$ et $285 \mathrm{~K}$, la largeur de la loi de diffusion associée aux mouvements 

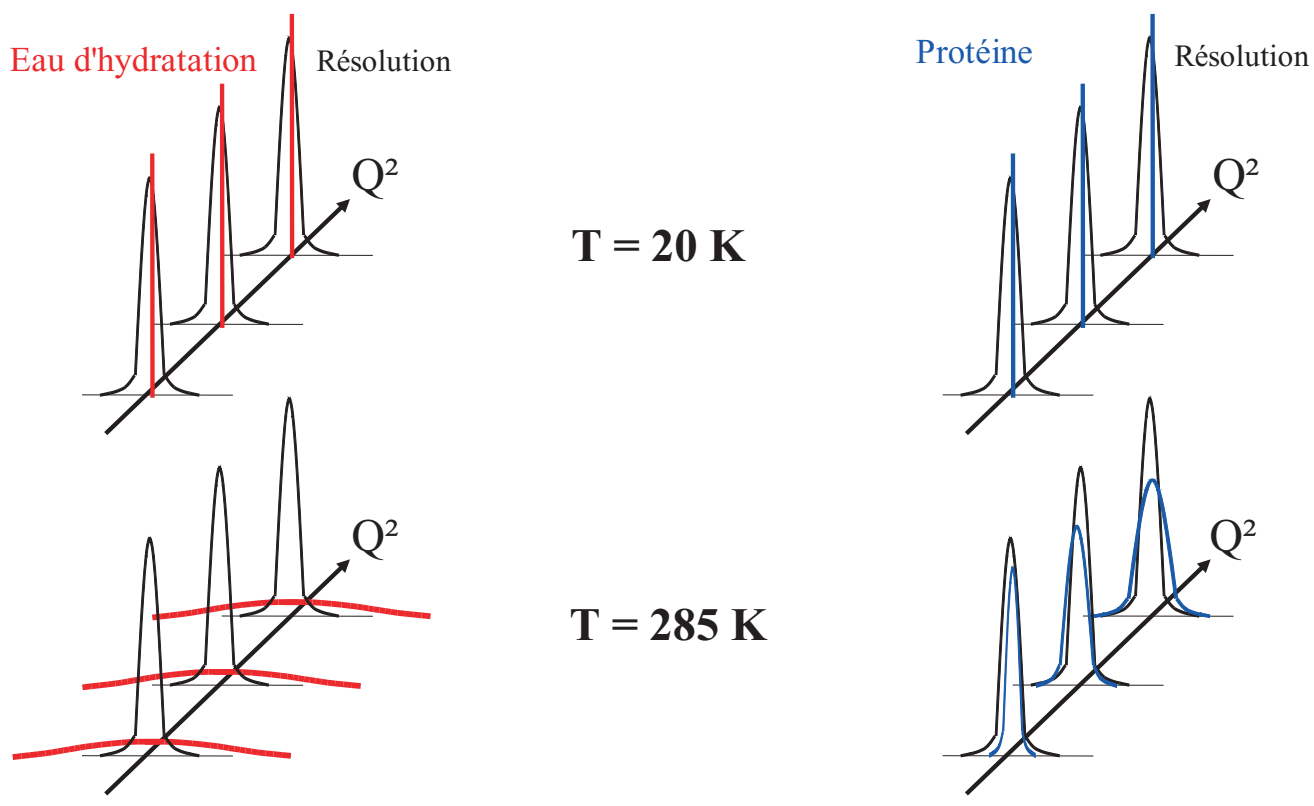

Figure 3. Schéma qualitatif des lois de diffusion des hydrogènes de la protéine et de l'eau d'hydratation à $20 \mathrm{~K}$ et à température ambiante par rapport à la résolution instrumentale.

de l'eau d'hydratation s'élargit, et devient plus grande que la largeur de la résolution en énergie de l'instrument. A $285 \mathrm{~K}$, l'intensité élastique des contributions quasi-élastiques de l'eau d'hydratation (second terme de l'équation (6)) est négligeable par rapport à l'EISF des hydrogènes de la protéine. En quelque sorte le signal de l'eau d'hydratation «sort » de la fenêtre élastique de l'instrument.

Sur la figure 3, la situation est résumée d'une manière assez qualitative-schématique. A $20 \mathrm{~K}$, les lois de diffusion théoriques sont superposées à la résolution instrumentale. L'eau d'hydratation et les hydrogènes de l'enzyme diffusent les neutrons de manière purement élastique. L'EI varie avec $\mathbf{Q}$ sous forme d'un Debye-Waller vibrationnel (les pics vibrationnels ne sont pas dessinés pour des raisons de clarté). A $285 \mathrm{~K}$, les lois de diffusion théoriques de l'eau et de l'enzyme contiennent des contributions élastiques et quasi-élastiques qui varient de manière différente avec $\mathbf{Q}$ et l'énergie: Bien que les hydrogènes de l'enzyme ne diffusent pas les neutrons de manière purement élastique, la loi de diffusion associée aux molécules d'eau d'hydratation s'élargit davantage par rapport à la résolution instrumentale et contribue peu à l'intensité élastique mesurée. Une interprétation quantitative des lois de diffusion sous-jacentes est en cours et ne sera pas détaillée ici.

\section{CONCLUSIONS}

Deux aspects de l'hydratation d'une protéine ont été étudiés par diffusion élastique incohérente de neutrons par l'exemple de la butyrylcholinestérase humaine: 1) Les relations entre la dynamique interne de l'enzyme et la composition ionique du solvant. 2) La dynamique de l'eau d'hydratation de la HuBChE en fonction de la température. Les résultats obtenus se résument de la manière suivante: 1) La présence du sel influence, en fonction de son type ionique, la dynamique interne de la protéine. Cet effet suit, pour les solvants étudiés, la série de Hofmeister: la présence du Tris- $\mathrm{HCl}$ (classé comme agent neutrechaotrope) ne modifie pas la dynamique interne de la protéine par rapport à l'absence totale d'ions dans l'eau d'hydratation. Par contre, la présence du phosphate de sodium (classé comme agent kosmotrope), réduit la flexibilité interne de la protéine (en termes de déplacements carrés moyens) au niveau d'un 
échantillon sec et supprime la transition dynamique. 2) La dynamique de l'eau d'hydratation à basse température (de 20 à $200 \mathrm{~K}$ ) ne se distingue pas de la dynamique interne de la protéine. Entre 200 et $260 \mathrm{~K}$, l'eau d'hydratation se mobilise par rapport aux atomes de la protéine et ne diffuse plus de manière purement élastique: l'eau « sort » de la fenêtre spatiale-temporelle de la résolution instrumentale. Au-delà de $260 \mathrm{~K}$ et jusqu'à $285 \mathrm{~K}$, le signal élastique de l'eau constitue un bruit de fond par rapport au signal élastique des atomes de la protéine et les MSDs mesurés dans cette gamme de température reflètent ainsi la dynamique interne de l'enzyme.

\section{Remerciements}

L'auteur tient à remercier tous les co-auteurs de [9], notamment Martin Weik et Giuseppe Zaccai, qui ont contribué de manière importante à ce travail. L'auteur remercie également Michel Ferrand pour les corrections du français.

\section{Références}

[1] Gabel F., Bicout D., Lehnert U., Tehei M., Weik M., Zaccai. G., Quart. Rev. Biophys. 35 (2002) 327.

[2] Lehnert U., Réat V., Weik M., Zaccai G., Pfister C., Biophys. J. 75 (1998) 1945.

[3] Tsai A., Neumann D., Bell L., Biophys. J. 79 (2000) 2728.

[4] Cordone L., Ferrand M., Vitrano E., Zaccai G., Biophys. J. 76 (1999) 1043.

[5] Fenimore P., Frauenfelder H., McMahon B., Parak F., Proc. Nat. Acad. Sci. USA 99 (2002) 16047.

[6] Tarek M., Tobias D., Phys. Rev. Lett. 88 (2002) 1381011.

[7] Touvier A., Xu J., Smith J., Biophys. J. 85 (2003) 1871.

[8] Grunwald J., Marcus D., Papier Y., Raveh L., Pittel Z., Ashani Y., J. Biochem. Biophys. Meth. 34 (1997) 123.

[9] Gabel F., Weik M., Doctor B., Saxena A., Fournier D., Brochier L., Masson P., Renault F., Silman I., Zaccai G., Biophys. J. 86 (2004) 3152.

[10] Ellman G., Courtney K., Andres V., Featherstone R., Biochem. Pharmacology 7 (1961) 88.

[11] Bée M., in Quasielastic neutron scattering. D. Millen (editor). Bristol and Philadelphia: Adam Hilger (1988).

[12] Guinier A., Fourmet G., in Small-Angle Scattering of X-rays. C. B. Walker (translator). New York, London: John Wiley \& Sons, Chapman \& Hall. (1955).

[13] Smith J., J. Quart. Rev. Biophys. 24 (1991) 227.

[14] Collins K., Washabaugh M., Quart. Rev. Biophys. 18 (1985) 323.

[15] Burgess S., Lister A., J. Prot. Chem. 7 (1988) 641. 